\title{
PESO AO NASCER E EXCESSO DE MORTALIDADE INFANTIL EM SÃO PAULO *
}

\author{
Carlos Augusto Monteiro **
}

Monteiro, C. A. Peso ao nascer e excesso de mortalidade infantil em São Paulo, Brasil. Rev. Saúde públ., S. Paulo, 14:1-8, 1980.

RESUmo: A influência que a distribuição do peso ao nascer exerceu sobre os coeficientes de mortalidade infantil de São Paulo em 1976 foi estudada através do ajuste destes coeficientes a uma distribuição ideal do peso ao nascer. Diferentemente de outros contextos de excessiva mortalidade, o excesso de óbitos infantis de São Paulo não parece dever-se fundamentalmente ao peso ao nascer. Algumas evidências sugerem que os fatores ate mortalidade do ambiente físico e social do municipio poderiam ser mais vulneráveis a af̧óes destinadas a reduzir a mortalidade infantil do que o seriam os fatores ligados à vitalidade do recém-nascido.

Unitermos: Peso ao nascer. Mortalidade infantil, S. Paulo, Brasil.

\section{INTRODUÇÃO}

Do mesmo modo que em outras cidades pertencentes ao chamado terceiro-mundo, na cidade de São Paulo o risco de óbito infantil em muito ultrapassa o mesmo risco observado em países desenvolvidos ${ }^{8}$. Esse excesso de mortalidade tornou-se particularmente notável nos últimos anos uma vez que, paralelamente a um período de inusitado crescimento econômico, São Paulo viuse às voltas com uma importante elevação de sua já alta mortalidade infantil ${ }^{1}$. Esta tendência crescente a tem colocado não somente em desigualdade com localidades desenvolvidas como, também, até mesmo com outras cidades da América Latina con- sideradas menos desenvolvidas economicamente ${ }^{4}$.

Entre as causas do excesso da mortaldade infantil de São Paulo, particular importância vem sendo atribuída às doenças infecciosas, com notável destaque para a doença diarréica, cuja taxa de mortalidade em São Paulo parece ser das mais altas do continente 4 . Outro aspecto relevante da mortalidade infantil de São Paulo, ao qual se tem atribuido parte do excesso de mortalidade, é o relativamente alto componente de mortalidade neonatal e a alta proporção de recém-nascidos de baixo peso

\footnotetext{
* Pesquisa financiada pela Fundação de Amparo a Pesquisa do Estado de São Paulo - FAPESP (Processo 78/1109).

* Do Departamento de Nutrição da Faculdade de Saúde Pública da Universidade de São Paulo - Av. Dr. Arnaldo, 715 - 01255 - São Paulo, SP - Brasil.
} 
Montelro, C. A. Peso ao nascer e excesso de mortalidade infantil em São Paulo, Brasil. Rev. Saude publ., S. Paulo, 14:1-8, 1980.

(peso ao nascer menor do que $2.500 \mathrm{gr}$ ) entre estes óbitos neonatais *

Quanto à doença diarréica, há muitas evidências de que esta desempenhe papel significativo no excesso de óbitos infantis de São Paulo e que sugerem, ainda, que investimentos no saneamento básico da cidade poderiam ter forte impacto na redução dos seus níveis de mortalidade ${ }^{t}$.

Já quanto ao baixo peso ao nascer, dado - caráter fundamentalmente associado de sua ação sobre o óbito infantil, e dada a total ausência de estudos populacionais que estimem sua incidência entre os recém-nascidos vivos, bastante mais difícil parece ser a evidenciação do seu papel no excesso de mortalidade infantil apresentado pelo município.

Estudos realizados em outros contextos com o objetivo de quantificar a participaçăo do peso ao nascer nos níveis da mortalidade infantil, tem invariavelmente concluido por uma elevada participação daquele fator.

Nos Estados Unidos, em 1960, o peso ao nascer seria o principal fator a determinar a duas vezes maior mortalidade infantil da sua população não branca ${ }^{15}$. Nas grandes cidades americanas, em 1970, igualmente demonstrou-se que o peso ao nascer explicaria a maior parte do diferencial de mortalidade existente entre os estratos pobres e não pobres da população não branca ${ }^{14}$. Seria ainda o baixo peso ao nascer que explicaria de "per si" a maior parte do excesso de mortalidade infantil do país americano diante de países de menor mortalidade como a Suécia e a Holanda ${ }^{13}$.

Estudos realizados em paises não desenvolvidos são bastante mais escassos. $\mathrm{Na}$ Guatemala, estudos longitudinais realizados pelo Instituto de Nutrición de Centroamérica y Panamá (INCAP) na sua zona rural vem demonstrando que também ali o peso ao nascer desempenharia importante papel na determinação dos níveis da mortalidade infantil. No pobre altiplano indigena, a performance do peso ao nascer seria responsável pela totalidade do excesso de mortalidade neonatal e por parte considerável do excesso de mortalidade infantil da região sobre a mortalidade de localidades desenvolvidas 9 .

Nas aldeias de população mestiça no leste da Guatemala, a simples redução da incidência do baixo peso ao nascer, via suplementação calórica a gestantes, teria se mostrado capaz de reduzir à quase metade os niveis da mortalidade infantil ${ }^{5}$. Esta última experiência, segundo seus autores, abriria um importante campo de ação para a Saúde Pública, em termos de reduzir coeficientes de mortalidade infantil em inúmeras comunidades pobres de todo o mundo ${ }^{\circ}$ e significaria que o grupo de gestantes deve representar a primeira prioridade para os programas de suplementação alimentar \%.

Estimulada sobretudo pela experiência guatemalteca e baseada nas características da mortalidade infantil de São Paulo, a Secretaria de Estado da Saúde de São Paulo passou, a partir de 1977 , a incluir na sua programação materno infantil a suplementação rotineira e indiscriminada das gestantes matriculadas nas unidades sanitárias do Estado 20. Tal atitude foi seguida também pelas unidades sanitárias do município que, assim como as unidades do Estado, passaram a distribuir no seu serviço de pré-natal o suplemento calórico-protéico denominado Gestal.

A preocupação fundamental deste trabalho será procurar quantificar a participação do peso ao nascer nos niveis de mortalidade infantil do município no ano de 1976 e a partir dela contribuir para uma discussão crítica em torno tanto das causas da elevada mortalidade infantil do municipio, como das expectativas de sua redução.

\section{MATERIAL E METODOS}

A caracterização da influência do peso ao nascer em São Paulo sobre sua mortalidade infantil foi conduzida através do ajuste desta mortalidade segundo uma dis- 
Monteiro, C. A. Peso ao nascer e excesso de mortalidade infantil em São Paulo, Brasil. Rev. Saúde pübl., S. Paulo, 14:1-8, 1980.

tribuição ideal de peso ao nascer. Utilizouse aqui o chamado ajuste indireto dos coeficientes de mortalidade que exige da população de estudo apenas o conhecimento dos coeficientes brutos de mortalidade e a composição da população quanto à variável a ser controlada ${ }^{23}$. O ajuste consiste na multiplicação do coeficiente observado de mortalidade por um fator de ajuste que leva em consideração apenas as diferenças de composição entre a população de estudo e a população de referência. $O$ fator de ajuste é obtido pela divisão do coeficiente de mortalidade observado na população de referência pelo coeficiente que seria regis- trado se esta população tivesse a mesma composição da população de estudo, o que exige, portanto, o conhecimento dos coeficientes específicos de mortalidade da população de referência.

A população de referência adotada foi a população branca dos Estados Unidos, cuja coorte de recém-nascidos do ano de 1960 apresenta confiáveis coeficientes de mortalidade infantil segundo peso ao nascer ${ }^{15}$. No caso, portanto, o coeficiente de mortalidade de São Paulo em 1976 ajustado segundo a distribuição do peso ao nascer de população branca dos Estados Unidos de 1960 seria dado pela fórmula:

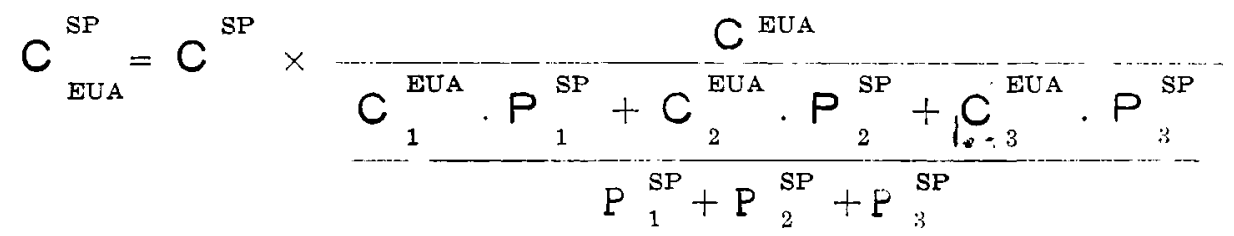

onde o coeficiente de mortalidade de São Paulo - $\mathrm{C}^{\mathrm{SP}}$ - é multiplicado pelo fator de ajuste que é a razão entre $o$ coeficiente americano $-\mathrm{C}^{\text {EUA }}-$ dividido pelo coeficiente que seria esperado se a ponderação entre seus coeficientes especificos de mortalidade por peso ao nascer fosse a distribuição do peso ao nascer de São Paulo em 1976. Os intervalos do peso de nascimento utilizados para os coeficientes especificos de mortalidade por peso ao nascer nos Estados Unidos $C_{1}^{\text {EUA }}, C_{2}^{\text {EUA }}, C_{3}^{\text {EUA }}$ - e para a distribuição percentual dos recém-nascidos vivos de São Paulo $-\mathrm{P}_{1}^{\mathrm{SP}}, \mathrm{P}_{2}^{\mathrm{SP}}$, $\mathrm{P}_{3}^{\mathrm{SP}}$ - foram respectivamente: menor ou igual a $2.500 \mathrm{gr}$, de 2.501 a $3.000 \mathrm{gr}$ e maior que $3.000 \mathrm{gr}$.

Os coeficientes de mortalidade infantil de São Paulo para 1976 referem-se apenas a nascimentos e óbitos de residentes em São Paulo 19. Seguindo recomendação técnica recente do Movimento do Registro Civil no Estado de São Paulo ${ }^{19}$, aos nascimentos de 1976 registrados naquele mesmo ano, foram somados os nascimentos de 1976 registrados no ano seguinte. Neste trabatho serāo estudados tanto os coeficientes de mortalidade infantil - número de óbitos de menores de 1 ano para cada mil nascidos vivos - como o coeficiente de mortalidade neonatal - número de óbitos de menores de 28 dias para cada mil nascidos vivos.

A distribuição do peso ao nascer para o município de São Paulo foi baseada no estudo de uma amostra probabilistica do total de nascimentos vivos ocorridos nas maternidades e hospitais do município em $1976^{11}$. A distribuição utilizada prevê uma margem de erro não maior que $1 \%$ para a estimação da proporção de recém-nascidos de baixo peso com um coeficiente de confiança de $95 \%^{11}$.

\section{RESULTADOS}

\section{$O$ ajuste da mortalidade infantil}

O coeficiente de mortalidade infantil do municipio de São Paulo em 1976 foi de 75,03 por mil nascidos vivos. A distribuição 
MONTEIRo. C. A. Peso ao nascer e excesso de mortalidade infantil em São Paulo, Brasil. Rev. Saude pübl., S. Paulo, 14:1-8, 1980.

do peso ao nascer dos seus recém-nascidos vivos foi estimada neste ano em 9,69\%, $28,07 \%$ e $62,24 \%$, respectivamente, para nascidos com $2.500 \mathrm{gr}$ ou menos, nascidos de 2.501 a $3.000 \mathrm{gr}$ e nascidos com mais de $3.000 \mathrm{gr}$.

A população de referência utilizada para o ajuste da mortalidade do município a uma distribuição ideal do peso ao nascer - a da coorte americana de recém-nascidos vivos da cor branca de 1960 - apresenta coeficiente global de mortalidade infantil de 22,17 e coeficientes específicos de 191,20, 17,32 e 7,97 , respectivamente, para nascidos com $2.500 \mathrm{gr}$ ou menos, nascidos entre 2.501 e $3.000 \mathrm{gr}$ e nascidos com mais de $3.000 \mathrm{gr}$.

$O$ ajuste indireto do coeficiente de mortalidade infantil de São Paulo de 1976 segundo a distribuição do peso ao nascer da coorte americana de referência

$$
\begin{gathered}
75,03 \times \frac{22,17}{191,20-9,69 \%+\frac{28,07 \%+7,97-62,24 \%}{100 \%}}= \\
=75,03 \frac{22,17}{28,35}=58,52
\end{gathered}
$$

levaria a mortalidade infantil de São Paulo de 75,03 para 58,52 por mil nascidos vivos. lsto é, mantidas as demais condiçōes de 1976, porém alterada a distribuição do peso de nascimento dos recém-nascidos de São Paulo de modo a igualá-la à observada entre os recém-nascidos brancos dos Estados Unidos, registrar-se-ia uma redução de $22 \%$ no coeficiente de mortalidade infantil do município de São Paulo. Desta forma, o diferencial de mortalidade infantil de São Paulo em relação à população branca dos Estados Unidos iria de $75,03-22,17=$ $=52,86^{\circ} / 00$ para $58,52-22,17=$ $=36,35 \%$ oo ou seja, as diferenças existentes na composição dos pesos de nascimento das duas populações explicariam $31 \%$ do excesso de mortalidade infantil de São Paulo.
Os restantes $69 \%$ deveriam ser procurados, portanto, em fatores outros que não o peso de nascimento.

\section{$O$ ajuste de mortalidade neonatal}

O coeficiente de mortalidade neonatal do município de São Paulo em 1976 foi de 32,93 por mil nascidos vivos. $\mathrm{Na}$ mesma população americana de referência ele foi de $16,88 \mathrm{com}$ coeficientes específicos de $177,38,10,07$ e 3,96 para nascidos com $2.500 \mathrm{gr}$ ou menos, nascidos entre $2.501 \mathrm{e}$ $3.000 \mathrm{gr}$ e nascidos com mais de $3.000 \mathrm{gr}$.

$O$ ajuste indireto do coeficiente de mortalidade neonatal de São Paulo em 1976 segundo a distribuição da coorte americana de referência

$$
\begin{gathered}
32,93 \times-\frac{177,38 \cdot 9,69 \%+10,07 \cdot 28,07 \%+3,96 \cdot 62,24 \%}{100 \%}= \\
=32,93 \cdot \frac{16,88}{22,48}=24,70
\end{gathered}
$$

levaria a mortalidade neonatal de São Paulo de 32,93 para 24,70 por mil nascidos vivos. Isto é, mantidas as demais condições de 1976, porém alterada a distribuição do peso de nascimento dos recém-nascidos de São
Paulo de modo a igualá-la à observada na população de referência, registrar-se-ia uma redução de $25 \%$ na mortalidade neonatal do município. Aqui, também da mesma forma, o diferencial da mortalidade neo- 
MONTEIRo, C. A. Peso ao nascer e excesso de mortalidade infantil em são Paulo, Brasil. Rev. Saúde pübl., S. Paulo, 14:1-8, 1980.

natal de São Paulo em relação à população branca dos Estados Unidos seria alterado de $32,93-16,88=16,05$ para $24,70-$ $16,88=7,82$, ou seja, neste caso as diferenças existentes na composição do peso de nascimento explicariam $51 \%$ do excesso de mortalidade neonatal de São Paulo, restando $49 \%$ que deveriam ser atribuídos a outros fatores que não o peso ao nascer.

\section{DISCUSSAO}

Em 1976, o coeficiente de mortalidade infantil de São Paulo foi de 75,03 por mil nascidos vivos. Este coeficiente denota um excesso de óbitos infantis quando comparado com os mesmos coeficientes de 1976 de paises altamente desenvolvidos como a Suécia e a Holanda, 8,7 e 10,5:2, ou mesmo quando comparado com os coeficientes de paises latinoamericanos como Cuba, 22,9 em $1976^{10}$, Panamá, 33,3 em $1973^{18}$, ou Costa Rica, 37,1 em 1975 9. Com 75,0 óbitos por mil nascidos vivos, a mortalidade infantil de São Paulo excede mesmo o coeficiente médio atribuído pela Organização Mundial de Saúde à região temperada da América do Sul, 62 óbitos por cada mil nascidos vivos ${ }^{13}$.

Em 1976, a incidência de recém-nascidos de baixo peso em São Paulo foi de 9,7\%11. A comparação desta incidência com a registrada em países desenvolvidos aponta uma situação também desfavorável para São Paulo. Bastante desfavorável quando comparada à da Suécia e Holanda, 5,0 e 5,5\%13, porém menos desfavorável quando comparada a dos Estados Unidos, 7,3\%12.

$A$ - comparação da incidência do baixo peso ao nascer de São Paulo com outras localidades do terceiro-mundo atribui, entretanto, posição favorável à São Paulo. Em uma revisão de estudos que abrangeram oito populações da América Latina e Asia T observaram-se incidências de 8 a $43 \%$, sendo que apenas em uma registrou-se incidência inferior à de São Paulo, tratandose neste caso de um estrato populacional de elevado nível sócio-econômico. A posição relativamente favorável de São Paulo em relação à incidência do baixo peso ao nascer confirma-se quando se observa que em Cuba, Panamá e Costa Rica, os países latino-americanos de mais baixas mortalidades infantis, aquela incidência é de $10,6 \%^{10}, 10,5 \%^{3}$ e $9,2 \%$.

Mesmo quando consideradas exclusivamente as maternidades públicas e filantrópicas, que teoricamente atendem à população mais pobre do município, a incidência do baixo peso ao nascer não ultrapassa os $12 \% 11$, distante, portanto, ainda, das cifras encontradas para os estratos populacionais mais pobres da América Latina e Asia que apresentam incidências que vão de 14 a $43 \%$ ?

Com 9,7\% de recém-nascidos de baixo peso, São Paulo coloca-se em posição bastante favorável em relação à incidència média estimada para a região temperada da América do Sul, $15 \%{ }^{17}$.

Para se estudar o papel que o peso estaria desempenhando no excesso de mortalidade infantil apresentado pelo município de São Paulo, utilizou-se como população de referência, a população branca dos Estados Unidos que em 1960 apresentou mortalidade infantil de 22,17 e incidência de baixo peso ao nascer de $6,8 \% 15$.

$\mathrm{O}$ ajuste da mortalidade infantil de São Paulo à distribuição de peso ao nascer da população branca americana mostrou-se capaz de reduzir aquela mortalidade de $75,03 \%$ para $58,52 \%$, revelando que $31 \%$ do excesso relativo de mortalidade de São Paulo poderiam ser atribuídos à atuação do peso ao nascer e os restantes $69 \%$ a fatores outros que não o peso ao nascer.

Se bem que ponderável, o papel desempenhado pelo peso ao nascer no excesso de óbitos infantis de São Paulo ainda pode ser considerado modesto se comparado ao papel do peso ao nascer nos grandes diferenciais americanos de mortalidade. Ali, ajustes semelhantes de mortalidade permi- 
Monteiro, C. A. Peso ao nascer e excesso de mortalidade infantil em são Paulo, Brasil. Rev. Saude puibl., S. Paulo, 14:1-8, 1980.

tem supor que a atuação do peso ao nascer responde por $63 \%$ do excesso de mortalidade infantil da população não branca ${ }^{15}$ e por $71 \%$ do excesso da população não branca e pobre ${ }^{14}$.

Igualmente mais eficazes do que em São Paulo seriam os ajustes da mortalidade infantil efetuados nos já reduzidos coeficientes de mortalidade do Panamá e Cuba. No primeiro caso, ajuste idêntico ao efetuado em São Paulo reduziria em $71 \%$ o excesso de mortalidade que em 1973 o Panamá apresentava em relação à população americana de referência. No segundo caso, o mesmo ajuste chegaria a inverter o diferencial de mortalidade, passando Cuba em 1976 a ostentar menor mortalidade do que a população branca dos Estados Unidos elm 1960.

No contexto da elevada mortalidade infantil da população indigena da Guatemala, também o papel do peso ao nascer parece mais importante. Ali, o mesmo ajuste da mortalidade à distribuição americana de peso ao nascer reduziria em $98 \%$ o excesso relativo de óbitos infantis.

Quanto ao componente neonatal da mortalidade infantil de São Paulo, o efeito do ajuste é mais eficaz, reduzindo em $51 \%$ o excesso de óbitos do município. Relativamente, entretanto, aos diferenciais americanos de mortalidade neonatal, novamente esta participação pode ser considerada pequena, uma vez que o controle de variável peso ao nascer explica praticamente $100 \%$ do excesso de óbitos neonatais dos estratos não-branco e não-branco pobre dos Estados Unidos 14,15. O mesmo se pode dizer tanto quanto à alta mortalidade neonatal da Guatemala como quanto às baixas mortalidades neonatais de Cuba e Panamá. Nestas três localidades, ajustes idênticos aos efetuados em São Paulo chegariam a inverter os diferenciais de mortalidade, fazendo com que as mesmas apresentassem coeficientes de mortalidade neonatal menores do que os da população branca americana de 1960 .
Esquematicamente divididos em um componente relativo à vitalidade do recém-nascido ao iniciar o primeiro ano de vida e em um componente relativo ao ambiente físico e social que vai envolvê-lo neste primeiro ano, pode-se dizer que os fatores do excesso de mortalidade ligados à vitalidade do recém-nascido desempenham em São Paulo um papel menos importante do que o fazem em outros contextos de excessiva mortalidade. Diferentemente do pequeno excesso da mortalidade infantil de Cuba e Panamá (22,9 e 33,3 por mil nascidos vivos), diferentemente do intermediário excesso da mortalidade infantil da população não-branca dos Estados Unidos (41,4 por mil nascidos vivos) e diferentemente do elevado excesso da mortalidade infantil da população indígena da Guatemala (95,3 por mil nascidos vivos), o exceso de mortalidade infantil de São Paulo parece dever-se mais a fatores ligados ao ambiente físico e social que envolve a criança menor de um ano do que propriamente à vitalidade com que esta inicia sua vida.

É altamente significativo que os recém-nascidos de São Paulo, que aqui nascem com peso inclusive superior ao dos recém-nascidos cubanos, panamenhos e não-brancos americanos, aqui tenham 2 a 3 vezes maior probabilidade de virem a morrer antes de completarem um ano.

É também altamente significativo que entre a população cubana e a população não-branca dos Estados Unidos, onde 0 peso ao nascer parece fundamental em determinar os seus excessos relativos de mortalidade infantil, a reduçāo destes excessos venha se fazendo independentemente do comportamento do peso ao nascer. De 1950 para 1967 a mortalidade infantil da população não-branca americana reduziu-se de 44,5 para 35,9 por mil nascidos vivos, enquanto a incidência de recém-nascidos de baixo peso chegou mesmo a subir de 10,2 para 13,6\%16,21. De 1970 a 1976 a mortalidade infantil de Cuba reduziu-se notavelmente, de 38,8 para 22,9 , rompendo o privilégio das baixas taxas de mortalidade 
MONTEIRo, C. A. Peso ao nascer e excesso de mortalidade infantil em São Paulo, Brasil. Rev. Saúde públ., S. Paulo, 14:1-8, 1980.

dos países desenvolvidos, enquanto a incidência de recém-nascidos de baixo peso foi de 10,3 para $10,8 \%{ }^{10}$.

Os comportamentos da mortalidade intantil e do peso ao nascer destas duas população e as características da mortalidade e do peso ao nascer dos recém-nascidos de
São Paulo estariam a sugerir que os fatores de mortalidade do ambiente físico e social, presentes em São Paulo, poderiam ser mais vulneráveis a ações determinadas a reduzir a mortalidade infantil do que os fatores de mortalidade ligados à vitalidade dos recém-nascidos.

Monteiro, C. A. [Birth weight and increased infant mortality in S. Paulo, Brazil.] Rev. Saúde públ., S. Paulo, 14:1-8, 1980.

ABSTRACT: Infant mortality in S. Paulo in 1976 was studied to find out just how much birth weight may influence infant mortality. Birth weight was found to be an important factor, but not as great as in other population groups. Evidence suggests that infant mortality in $S$. Paulo can be reduced by actions against factors influencing physical and social environment, rather than against factors linked to newborn vitality.

UNITERMS:Birth weight. Infant mortality, S. Paulo, Brazil.

\section{REFERENCIAS BIBLIOGRAFICAS}

1. CAMARGO. C. P. F, et al. São Paulo, 1975: crescimento e pobreza. 4" ed. São Paulo, Edições Loyola, 1976.

2. DELGADO, H. et al. Aplicación de técnicas simplificadas a programas de salud maternoinfantiles: los aspectos nutricionales de alto riesgo. Bol. Ofic. sanit. panamer., 84:295-305, 1978.

3. DEMOGRAPHIC YEARBOOK. (United Nations) New York, 1975. p. 753.

4. LAURENTI, R. Alguns aspectos particulares referentes aos resultados da. Investigação Interamericana de Mortalidade na Infância na área do projeto de São Paulo. Bol. Ofic. sanit. panamer., $89: 1-14,1975$.

5. LECHTIG, A. et al. Os efeitos de uma nutrição aperfeiçoada a partir da concepçăo da criança até os 3 anos de idade na mortalidade infantil. $J$. Pediat., 42:40-7, 1977.

6. LECHTIG, A. et al. Effect of food supplementation during pregnancy on birth welght. Pediatrics, 56:508-20, 1975.
7. LECHTIG, A. et al. Maternal nutrition and fetal growth in developing societies. Socioeconomic factors. Amer. J. Dis. Child., 129:434-7, 1975.

8. LESER, W. Relacionamento de certas caracteristicas populacionais com a mortalidade infantil no município de São Paulo de 1950 a 1970. Probl. bras., $10(109): 17-31,1972$.

9. MATA, L. et al. Implicaciones del bajo peso al nacer para la salud publica. Arch. lat.-amer. Nutr., 27 (supl. 1):198-222, 1977.

10. MINISTERIO DE SALUd DE CUBA. Informe anual:197\%. Havana, 1978.

11. MONTEIRO, C. A. O peso ao nascer no municipio de São Paulo: impacto sobre os niveis de mortalidade na infancia. São Paulo, 1979. [Tese de Doutoramento - Faculdade de Saúde Pública da USP].

12. NATIONAL CENTER FOR HEALTH STATISTICS. Final natality statistics, 1977. Mth. vital Statist. Rep., 27 (Suppl.) Feb. 1979. 
Monteiro, C. A. Peso ao nascer e excesso de mortalidade infantil em São Paulo, Brasil. Rer. Saúde pübl. S. Paulo, 14:1-8, 1980.

13. NATIONAL CENTER FOR HEALTH STATISTICS. International comparison of perinatal and infant mortaluty the United States and six West Europeaul Countries. Vital Hlth Statist. Ser. 3. (6) 1967.

14. NATIONAL CENTER FOR HEALTH STATISTICS. Selected vital and health statistics in poverty and nonpoverty areas of 19 large cities. United States, 1969-1971. Vital Hlth Statist. Ser. 21, (26) 1975 .

15. NATIONAL CENTER FOR HEALTH STATISTICS. A study of infant mortality from linked records by birth weight. period of gestation, and other variables. United States, 1960, liverbirtin cohort. Vital Hith Statist, Ser. 20, (12) 1972.

16. NATIONAL CENTER FOR HEALTH STATISTICS. Trends in "prematurity": United States, 1950-67. Vital Hlth Statist. Ser. 3, (15) 1972.

17. PETROS - EARVAZIAN. A, \& BEHAR. I Low birth weight: a major global problem. In: Sterky. G. \& Mellander, L. Buth weight distmbution: an indicator of social derelopment. Uppsala Swedish Agency for Research Cooperation with Dereloping Countries, 1978. (Report. R. 2).
18. SATED, A. Memoria apresentada al Exmo. Señor Presidente de la Republica $y$ a la Asamblea de Representantes de Corregimientos. Panamá, 1976.

19. SECRETARIA DE ECONOMIA E PLANEJAMENTO. Movimento do registro cirll no Estado de São Paulo: 19ít a 1976. São Paulo, 1977. (Estudos e Pesquisas, 12).

21. SUPLEMENTAÇÃO alimentar a gestantes para reduzir coeficiente de mortalidade infantil. Saúde, S. Paulo, 1(2): 1, 1976 .

21. WEGMAN. M. E. Annual summary of vital statistics. 1973. Pedatrics, 54: $677-81,1974$.

22. WEGMAN, M, E. Annual summary of rital statistics: 1976. Pediatrics, 60:797-804. 1977.

2.) WORLD HEALTH ORGANIZATION, Manual of mortality analysis. A manual on methods of analysis of national mortality statistics for public health purposes. Genera. 1977.

Recebido para publicação em 18/06/1979 Aprovado para publicasão em so/10/1979 